M Compte-Pujol, K Matilla, S Hernández (2018): "Estrategia y Relaciones Públicas: un estudio bibliométrico comparativo”. Revista Latina de Comunicación Social, 73, pp. 748 a 764. http://www.revistalatinacs.org/073paper/1280/39es.html

DOI: $10.4185 / R L C S-2018-1280$

\title{
Estrategia y Relaciones Públicas: un estudio bibliométrico comparativo
}

\author{
Strategy and Public Relations: a Bibliometric \\ Comparative Study
}

\author{
Marc Compte-Pujol [CV] [ (DORCID] [ $\mathrm{B}$ GS] Profesor asociado de la Facultad de Empresa y \\ Comunicación. Universidad de Vic-Universidad Central de Cataluña (España) - \\ marc.compte@uvic.cat
}

Kathy Matilla [CV] [ ORCID] [ 8 GS] Profesora asociada de la Facultad de Comunicación y Relaciones Internacionales Blanquerna. Universidad Ramón Llull (España) kathyms@blanquerna.url.edu.

Salvador Hernández [CV] [ [DPRCID] [ ${ }^{8}$ GS] Profesor titular de la Facultad de Ciencias Sociales y de la Comunicación. Universidad Católica San Antonio de Murcia (España) shernandez@ucam.edu

\begin{abstract}
s
[ES] Este artículo presenta un estudio bibliométrico de las revistas de relaciones públicas catalogadas en las bases de datos Social Sciences Citation Index-Communication-Journal List y de las de estrategia del Social Sciences Citation Index-Management-Journal List, ambas propiedades de Thomson Reuters hasta octubre de 2016. Mediante análisis de contenidos se identifican los artículos que tratan la estrategia en las revistas especializadas en relaciones públicas y al revés: las especializadas en estrategia que contemplan las relaciones públicas en sus contenidos. Las variables de análisis fueron los periodos temporales en que se habían publicado mayor número de artículos; cuestiones de género sobre los autores de los artículos analizados; número prevalente de autores por artículo; y los títulos de los artículos. Los resultados revelan que la estrategia aparece ampliamente tratada en las revistas de relaciones públicas, mientras que las relaciones públicas tienen presencia casi nula en las revistas de gestión especializadas en estrategia.

[EN] This article presents a bibliometric study of journals specialized in public relations (PR) that were listed in the Social Sciences Citation Index-Communication-Journal List database and journals specialized in strategy management that were listed in the Social Sciences Citation IndexManagement-Journal List. Both lists owned by Thomson Reuters until October 2016. Articles referring to the concept of "strategy" in PR journals were identified through content analysis, as well as those articles that included the concept "public relations" in journals specialized in strategy management. Variables of analysis were: periods of time for publishing; gender of authors; prevalent
\end{abstract}


number of authors per article; and titles of the articles. Results showed that strategy appeared widely treated in the journals specialized in PR, while PR had almost no presence in magazines specialized in strategy management.

\section{Keywords}

[ES] Bibliometría; Estrategia; Relaciones Públicas (RP); Social Sciences Citation Index (SSCI); Web of Science (WoS).

[EN] Bibliometrics; Public Relations (PR); Social Sciences Citation Index (SSCI); Strategy; Web of Science (WoS).

\section{Contens}

[ES] 1. Introducción. 2. Marco teórico. 3. Preguntas de investigación. 4. Metodología. 5. Limitaciones metodológicas. 6. Resultados. 6.1. Resultados de los términos "strategy" y "strategic" en las revistas de relaciones públicas. 6.1.1. Public Relations Review. 6.1.2. Journal of Public Relations Research. 6.2. Resultados de los términos "public relations" y "PR" en las revistas de estrategia. 6.2.1. Revistas “strategy”. 6.2.2. Revistas "strategic”. 7. Discusión y conclusiones. 8. Referencias bibliográficas.

[EN] 1. Introduction. 2. Theoretical framework. 3. Research questions. 4. Methodology. 5. Limitations. 6. Results. 6.1. Results of the terms "strategy" and "strategic" in journals specialized in Public Relations. 6.1.1. Public Relations Review. 6.1.2. Journal of Public Relations Research. 6.2. Results of the terms "public relations" and "PR" in the journals specialized in Strategy. 6.2.1. Journals with the term "Strategy". 6.2.2. Journal with the term "Strategic". 7. Discussion and Conclusions. 8. List of References.

Traducción de resumen de M. Comte-Pujol (Profesor asociado / Universidad de Vic-Universidad Central de Catalunya)

Traducción de artículo de Yuhanny Henares (Traductora académica, Universitat de Barcelona)

\section{Introducción}

La bibliometría consiste en la aplicación de técnicas cuantitativas al estudio de las características bibliográficas de los documentos (Castillo \& Xifra, 2006: 147).

La literatura especializada en bibliometría es abundantísima. Destacan, entre otros muchos, los siguientes autores: Beniger (1990); Bornmann y otros (2008); Brody y otros (2007); Bunz (2005); Burnham (1990); Butler (2008); Campbell (2008); Clarke (2007); Cope \& Kalantzis (1993, 2010); Cope, Kalantzis \& Magee (2011); Cope \& Phillips (2013); Craig \& Ferguson (2013); Davies (2009); De Solla Price (1965); Dewatripont y otros (2006); Edlin \& Rubinfeld (2004); Evans (2008); Ferreira et al. (2016); Fink \& Bourne (2007); García-Guinea \& Sota Rius (1998); Ginsparg (2007); González Quirós \& Martín (2009); Guédon (2001); Hannay (2007); Harnad (2009); Harzing \& Van Der Wal (2008); Jakubowicz (2009); Jefferson, Wager \& Davidoff (2002); Judson (1994); Kousha \& Thelwall (2007); Koehler (2001); Lee \& Bero (2006); López Baena y otros (2005); López Piñero (1972); Mabe \& Amin (2002); Mccabe y otros (2006); Meho, 2007; Morris (2009); Noyons y oOtros (2009); Norris \& Oppenheim (2007); Osca-Lluch y otros (2009); Pauly \& Stergiou (2008); Pritchard (1969); Schroeder (2007); Simons (2008); Stanley, 2007; Suber, 2007; Tenopir \& King (2009); Todd \& Ladle (2008); Van De Sompel \& Lagoze (2007); Van Leeuwen (2001); Wilbanks (2007); o Willinsky (2006). 
Los estudios bibliométricos:

permiten conocer los ámbitos en los que se desarrollan las temáticas de un campo científico, conocer las tendencias de las investigaciones, identificar a los grupos de investigación (investigadores) que están trabajando, verificar el grado de interconexiones internacionales entre investigaciones, la relación entre género, entre investigadores, los sistemas de citas de otras publicaciones, las autocitas de las publicaciones, las autocitas de los investigadores, los centros de investigación en los que se realizan (educativos, profesionales, investigadores). Es decir, todo un conjunto de parámetros que permiten establecer un panorama del estudio de la investigación en un campo concreto (Castillo \& Carretón, 2010: 293).

La disrupción de Internet y las redes sociales, así como la digitalización de los textos, la construcción de las infraestructuras open access y el crecimiento del número de repositorios, coincidentes grosso modo con el inicio del siglo XXI, ha acrecentado el interés y el debate sobre la creación y el intercambio de la producción científica (Cope \& Kalantzis, 2009: n/e).

Con el tiempo la producción bibliométrica se ha especializado y acotado su objeto de estudio. Así, se han identificado artículos sobre comunicación (So, 1988; Paisley, 1989; Cáceres \& Caffarel, 1993; Jones, 1997; Lauf, 2005; Lievrouw, 1990; Martínez Nicolás, 2009; Castillo \& Carretón, 2010; Castillo \& Rubio, 2010; Castillo-Esparcia et al., 2012; Roca-Correa \& Pueyo-Ayhan, 2012; Martínez-Nicolás \& Saperas-Lapiedra, 2016; Compte-Pujol et al., 2016) y también estudios específicos sobre relaciones públicas (Pasadeos \& Renfro, 1989, 1992; Pasadeos et al.; Sallot et al., 2003; Xifra \& Castillo, 2006; Ki \& Shin, 2006; Castillo \& Xifra, 2006; Pasadeos et al., 2010; Fussell et al., 2013; Míguez et al., 2014; Kim et al., 2014; Marca et al., 2014; Míguez-González et al., 2016; entre otros).

En octubre de 2016, Thomson Reuters hizo efectiva la venta de sus negocios de propiedad intelectual y ciencias a varios fondos de inversión afiliados con Onex Corporation y Baring Private Equity Asia. De este modo, la plataforma que con Thomson Reuters era conocida como Web of Knowledge (WoK), formada por un amplio catálogo de bases de datos bibliográficos, citaciones y referencias de publicaciones científicas sobre cualquier disciplina del conocimiento (científico, tecnológico, humanístico y sociológico), cambia su denominación a Clarivate Analytics. Una nueva compañía que seguirá incluyendo marcas como Web of Science (WoS), Cortellis, Thomson Innovation, Derwent World Patents Index, Thomson CompuMark, MarkMonitor, Thomson IP Manager y Techstreet, entre otras.

Dado el prestigio académico logrado por la WoK y la base de datos WoS en los últimos tiempos (Cope \& Kalantzis, 2009: n/e), los investigadores de los cinco continentes procuran publicar sus trabajos en las revistas incluidas en sus bases de datos (McNutt, 2014), por lo que la plataforma se constituye en el más destacado escaparate que permite observar los principales temas objeto de estudio en cada disciplina y periodo, así como su evolución a través del tiempo y desarrollar estudios comparativos.

\section{Marco teórico}

Según Matilla y otros (2015), el término “relaciones públicas” (RP) aparece documentado por primera vez en los Estados Unidos de América en 1882 (Goldman, 1948: 2; Chaumely \& Huisman, 1963: 8; Cutlip, 1995: 208) y, en Europa, se empleó por primera vez en Alemania en 1937 (Nessmann, 1995: 151-160). Sus prácticas en los antiguos países comunistas se remontan a 1989 (Moloney, 2000: 40).

Las RP, tanto desde su orientación académica como profesional, se consideran un área funcional independiente en la literatura especializada y esta categorización directiva de la praxis está 
directamente vinculada a su orientación estratégica (Prat Gaballí, 1958; Matrat, 1970: 27, 37-49; Arnaldi, 1971: 217; Ugeux, 1973: 32-33; Ledigham \& Bruning, 2000; Van Ruler, 2000; Van Ruler y otros, 2000, 2004; Van Ruler \& Verčič, 2002, 2004).

Desde el punto de vista de la aplicación a la gestión y a la administración de empresas, en la edición de 2011 del macroestudio ECM-European Communication Monitor (http://www.zerfass.de/ECMWEBSITE/ECM-2011.html) se revela que la participación de los profesionales de las RP y la comunicación en los niveles directivos o la posibilidad de rendir cuentas al CEO-Chief Executive Officer son aspectos clave a la hora de valorar la responsabilidad jerárquica y el poder que recibe el departamento desde la alta dirección. El 60\% del total de los participantes del ECM-2011 declaró reportar directamente al CEO y un $17 \%$ a otro miembro del consejo de administración. Un 17,80\% de los directivos europeos pertenecía al comité de dirección. El 41,7\% de los encuestados europeos tenía responsabilidad sobre la totalidad de la gestión de la comunicación y de la relación con los stakeholders (grupos de interés). La mayoría (55\%) declaró ser responsable de, al menos, tres campos de la comunicación, mientras que un 10\% se limitaba a las relaciones con los media y a la comunicación externa. El 87\% de los participantes españoles consideró que el área funcional de la comunicación jugaba un papel importante en el rendimiento global de su organización, al generar activos financieros e inmateriales (70\% en España; 47,9\% en el resto de Europa). Casi el 70\% de los participantes españoles del ECM-2011 consideraba que el reto principal al que debía hacer frente el colectivo profesional era la evolución digital y la web social -tema que había crecido 30 puntos en los cinco años precedentes-, seguido de una mayor vinculación de las estrategias de comunicación con las estrategias de negocio (58\%), los nuevos métodos de evaluación (29\%) y la globalización (26,1\%).

El estudio británico State of the Profession Survey (2014) del Chartered Institute of PR-CIPR (http://www.cipr.co.uk/content/about-us/our-organisation), muestra que, en el Reino Unido, un 70\% de los 2.531 participantes consideró que la función esencial de sus tareas se centraba en el diseño y la ejecución del plan estratégico anual de comunicación, que debe aportar valor a las organizaciones.

Desde la perspectiva directiva de las RP y, por lo tanto, estratégica, nos interesaba conocer cual era la producción científica indizada en el WoS, e identificar los artículos que trataban la estrategia en las revistas especializadas en RP y, paralelamente, los de las revistas de gestión especializadas en estrategia que aplicaban las RP en sus contenidos y comprobar si existían alineamientos o divergencias entre ambas y, asimismo, consistencias con la literatura de referencia.

\section{Preguntas de investigación}

El objetivo principal de la investigación fue doble: (1) averiguar si en la muestra de las revistas de RP objeto de estudio se planteaba la perspectiva estratégica de la disciplina, y (2) si en las revistas de gestión especializadas en estrategia se publicaban contenidos específicos sobre RP, entre el 01.01.00 y el 15.07.15 en todos los casos.

Como objetivos secundarios se planteó conocer: (a) los años en que se habían publicado mayor número de artículos; (b) cuestiones de género sobre los autores de los artículos analizados; (c) número de autores por artículo; y (d) los títulos de los artículos, para identificar los temas tratados.

Se formularon doce (12) preguntas de investigación para dar respuesta a diversas variables de análisis que permitiesen el logro de dichos objetivos:

(PI1) ¿Cuáles son las revistas catalogadas en la base de datos SSCI-CommunicationJournal List del WoS especializadas en RP?

(PI2) ¿Cuáles son las revistas catalogadas en la base de datos SSCI-Management-Journal List del WoS especializadas en estrategia? 
(PI3) ¿Se contempla la perspectiva estratégica en los artículos publicados en las revistas especializadas en RP?

(PI4) ¿Se contemplan las RP en los artículos publicados en las revistas de gestión especializadas en estrategia?

(PI5) ¿Cuáles son los años en que se han publicado un mayor número de artículos en las revistas especializadas en $\mathrm{RP}$ ?

(PI6) ¿Cuáles son los años en que se han publicado un mayor número de artículos en las revistas especializadas en estrategia?

(PI7) ¿Cuál es la distribución de género de los autores de los artículos en las revistas especializadas en RP?

(PI8) ¿Cuál es la distribución de género de los autores de los artículos en las revistas especializadas en estrategia?

(PI9) ¿Cuál es el número de autores por artículo prevalente en las revistas especializadas en $\mathrm{RP}$ ?

(PI10) ¿Cuál es el número de autores por artículo prevalente en las revistas especializadas en estrategia?

(PI11) ¿Cuáles son los títulos de los artículos de las revistas especializadas en RP?

(PI12) ¿Cuáles son los títulos de los artículos de las revistas especializadas en estrategia?

\section{Metodología}

Para la selección de la muestra de las revistas especializadas en RP se buscó entre el universo de las setenta y ocho (78) revistas catalogadas en la base de datos SSCI-Communication-Journal List las que, en su cabecera o título incluyesen las palabras "public relations” o "PR”. Se identificaron dos (2) revistas cumplidoras del requisito: Public Relations Review (también indizada en Scopus) y Journal of Public Relations Research, sobre las cuales "Many previous meta-analyses studied that examined research trends in public relations exclusively employed these two as their sample” (Seon-Kyoung \& I-Huei, 2012: 71).

Se procedió de idéntica forma con el universo del ciento ochenta y ocho (188) revistas del SSCIManagement-Journal List en cuyo título o cabecera figurasen los términos "strategy" o "strategic". Once (11) revistas cumplían esas condiciones y se añadieron a la muestra: Business Strategy and the Environment; Global Strategy Journal; Journal of Economics Management Strategy; Journal of Family Business Strategy (n=4 para el término "strategy"); Advances in Strategic Management-A Research Annual; International Journal of Strategic Property Management; Journal of Strategic Information Systems; Strategic Entrepreneurship Journal; Strategic Organization; Strategic Management Journal; y Technology Analysis \& Strategic Management ( $\mathrm{n}=7$ para el término "strategic").

Todas las búsquedas se llevaron a cabo entre el 20 y el 23 de julio de 2015.

Tras identificar las revistas se llevó a cabo una búsqueda a través de los buscadores específicos de cada una de sus páginas web oficiales. Se establecieron parámetros de búsqueda en el título del artículo, en los resúmenes (abstracts) y en las palabras clave (keywords). Dichos parámetros fueron “strategy” y 
“strategic" para las revistas especializadas en RP -no hemos contemplado palabras derivadas de las mismas, como "strategically" o "strategies", a pesar de que en determinados casos también aparecían en los artículos analizados-, y “public relations” y "PR" para las revistas especializadas en estrategia en la revista Journal of Family Business Strategy se identificó en diversas ocasiones el parámetro "PR". Comprobando que se trataba del acrónimo "Perfomance Related” los artículos se desestimaron, no incluyéndose en el estudio-.

El parámetro temporal de búsqueda se acotó al periodo comprendido entre el 1 de enero de 2000 y el 15 de Julio de 2015. El inicio de la acotación se justifica porque la literatura especializada sitúa en los albores del nuevo milenio el más amplio desarrollo del interés académico y profesional por la praxis estratégica de las RP y porque, desde la perspectiva bibliométrica, es también ahí donde se sitúa la incorporación masiva a la era digital y a la digitalización de las revistas académicas (Cope \& Kalantzis, 2009: $\mathrm{n} / \mathrm{e})$.

Las búsquedas de las unidades de análisis se realizaron desde la web del WoS, en lengua inglesa para los términos de las variables de análisis, ya que "the role of English is the major language of modern day science" (Van Leeuwen, 2001: 344), de modo que "In 1995, for example, English made up over 95\% of publications in the Science Citation Index" (Tardy, 2004: 250), constituyéndose en la lingua franca (Yukio, 1992: n/e). Una vez determinadas las revistas, las siguientes búsquedas se realizaron desde sus respectivas webs corporativas, también en inglés. Posteriormente se depuraron los listados de artículos, eliminando duplicidades.

Mediante análisis de contenidos (Krippendorff, 2004; Wimmer \& Dominick, 1996) las variables de análisis de los artículos se trasladaron a una plantilla diseñada ad hoc, siendo éstas: (a) nombre de la revista; (b) número del volumen, del número y año de publicación, (c) nombre y apellido de los autores, (d) género de los autores, y (e) título del artículo. Se seleccionaron siguiendo metodología y parámetros bibliográficos inspirados en Koehler (2001); Castillo \& Xifra (2006); Castillo \& Carretón (2010); y Marca y otros (2014).

Koehler apunta en su investigación que el 62\% de los artículos están escritos por un único autor, seguido por dos autores (26\%), tres autores (8\%) y más de cuatro (4\%). Sin embargo, esta tendencia ha ido modificándose a lo largo de los años ya que en los 50 la media era de 1,2 autores por artículo y en los 90 pasó a 1,8 (Koehler, 2001: 122, Cfr. Castillo \& Carretón, 2010: 299).

El número de autores que participan en los artículos asciende a 378 de los cuales, un 53,7\% corresponde a autores y un 46,3\% a autoras. En la variable del género de los autores existe una diferencia de 7,4 puntos porcentuales: los hombres aparecen con mayor frecuencia que las mujeres en las revistas científicas de comunicación. Si tenemos en cuenta el género de los autores y el número de firmantes en los artículos, los resultados evidencian que son los hombres los que prefieren escribir artículos en solitario. De los 203 firmantes masculinos, el 52,71\% lo hacen de esta forma y el 23,15\% lo hace en colaboración con otro. En cuanto a las mujeres, escribir un artículo como únicas firmantes o en colaboración con otro autor muestra una diferencia menor que en el caso de los hombres. De las 175 autoras, el 41,7\% responde a artículos de una sola autora, frente a un 32,57\% de artículos con dos autoras. Los artículos con tres o más firmantes coinciden, desde el análisis del género, sin presentar diferencias significativas (Castillo \& Carretón, 2010: 310).

La suma de los trabajos firmados hasta por dos autores responde al 90,2\% de los artículos [...] El 25,5\% de los artículos de hasta dos autores se concentran en los de 1 a 8 páginas [...] Los artículos firmados por tres autores suponen el 5,1\%, [...]. Aquellos trabajos 
firmados por más de 3 autores no obtienen una representatividad significativa en el global analizado (Castillo \& Carretón, 2010: 317).

\section{Limitaciones metodológicas}

Las webs de la totalidad de las revistas permiten visualizar las variables de análisis de la investigación, excepto la revista Journal of Public Relations Research, que no ofrece las palabras clave de sus artículos. En las webs de las revistas Public Relations Review y Strategic Management Journal los nombres de pila de los autores únicamente se consignan con sus iniciales, lo que obligó a realizar una búsqueda suplementaria individual de cada uno de ellos a través de los buscadores Google y Google Academics (Kousha \& Thelwall, 2007; Schroeder, 2007; Harzing \& Van Der Wal, 2008) para completar las cuestiones de género [ver Columna 2 de Tabla 1, Tabla 2 y Tabla 6].

\section{Resultados}

En el análisis descriptivo del doble estudio los resultados se presentan incluidos en tablas de elaboración propia y con descripción escrita -numérica y porcentual- para facilitar su comprensión.

Las tablas pueden ser consultadas en Internet mediante sus respectivos enlaces que se muestran a continuación:

Tabla 1. El término "strategy" en Public Relations Review. https://issuu.com/marc532/docs/tabla_1_5961b28fce4543

Tabla 2. El término "strategic” en Public Relations Review. https://issuu.com/marc532/docs/tabla_2_9aa1838d09af53

Tabla 3. El término “strategy” en Journal of Public Relations Research.

https://issuu.com/marc532/docs/tabla_3

Tabla 4. El término "strategic” en Journal of Public Relations Research.

https://issuu.com/marc532/docs/tabla_4

Tabla 5. Los términos “public relations” y "PR” en las revistas “strategy”.

https://issuu.com/marc532/docs/tabla_5

Tabla 6. Los términos “public relations” y "PR” en las revistas "strategic”.

https://issuu.com/marc532/docs/tabla_6

\subsection{Resultados de los términos "strategy" y "strategic" en las revistas de relaciones públicas} 6.1.1. Public Relations Review

Tras eliminar 12 duplicidades, se identificaron 207 artículos válidos para el estudio, de los cuales 102 (49,27\%) cumplían con los requisitos de búsqueda “strategy” y 105 (50,72\%) con "strategic”.

Se contabilizaron un total de 412 autores (media de 1,99 autores/artículo). Los 102 artículos “strategy” fueron escritos por 203 autores, de los cuales 94 (46,30\%) eran hombres y 109 (53,69\%) mujeres. Los 105 artículos "strategic" salieron de las manos de 209 (50,72\%) autores, de los cuales 82 (39,23\%) 
eran hombres y 127 (60,76\%) pertenecían al género femenino. Del total de 207 artículos, pues, 176 se debieron a autoría masculina (42,71\%) frente a los 236 (57,28\%) de la mayor autoría femenina.

Son prevalentes los artículos "strategy" ( $\mathrm{n}=102)$ escritos por 2 autores $(48-47,05 \%)$, seguidos de los 34 (33,33\%) artículos escritos por 1 autor, 15 (14,70\%) escritos por 3 autores, 3 (2,94\%) escritos por 4 autores y 2 (1,96\%) los de 5 autores. En cuanto a los artículos "strategic" $(n=105)$ son mayoritarios los escritos por 1 autor (48-45,71\%), seguidos de los 35 (33,33\%) artículos escritos por 2 autores, 15 $(14,28 \%)$ escritos por 3 autores y 7 (6,66\%) cuya autoría se debe a 4 personas.

En el periodo analizado se publicaron artículos "strategy" y "strategic" que cumplían con los criterios de búsqueda todos los años, excepto en 2001, en que no se publicó ningún artículo "strategy” y en 2006, en que no se publicó ningún artículo con el parámetro "strategic”.

En los artículos "strategy" (n=102) en 2000 y 2003 se publicó un único artículo (0,98\%); en 2002 se publicaron 2 (1,96\%); en 2004, 4 (3,92\%); en 2006 y 2007, 5 (4,90\%); en 2005 y 2011, 6 (5,88\%); en 2010, 7 (6,86\%); en 2009 y 2013, 8 (7,84\%); en 2015 (hasta 15.07.15), 9 (8,82\%); en 2008, 12 (11,76\%); en 2014, 13 (12,74\%); y en 2012, 16 (15,68\%), siendo pues 2012 el año en que se publicaron más artículos “strategy” (15,68\%).

En los artículos “strategic" (n=105) se publicó únicamente 1 (0,95\%) artículo en 2001; 2 (1,90\%) en 2008; 3 (2,85\%) en 2002 y 2003; 4 (3,80\%) en 2000; 5 (4,76\%) en 2004 y 2015 (hasta el 15.07.15); 6 (5,71\%) en 2007 y 2011; 7 (6,66\%) en 2010; 8 (7,61\%) en 2005 y 2013; 15 (14,28\%) en 2009 y 2012; y 18 (17,14\%) en 2014 que es, pues, el año en que se publicaron más artículos con el criterio de búsqueda "strategic" $(17,14 \%)$.

Ver los títulos de los artículos en Tabla 1 (“strategy”) y Tabla 2 (“strategic”).

\subsubsection{Journal of Public Relations Research}

Se identificaron un total de 46 artículos que se adaptaban al criterio de búsqueda: 28 (60,86\%) “strategy" y 18 (39,13\%) "strategic", tras depurar duplicidades.

Los 46 artículos fueron escritos por un total de 95 autores (media de 2,06 autores/artículo). De los 50 autores (52,63\%) “strategy”, 19 (38,00\%) fueron escritos por hombres y 31 (62,00\%) por mujeres. De los 45 (47,3\%) autores “strategic”, 24 (53,33\%) fueron de autoría masculina y 21 (46,66\%), femenina. Los 28 artículos "strategy" fueron mayoritariamente escritos por 1 autor (13-46,42\%) y los 18 artículos "strategic" fueron escritos prevalentemente por 2 autores (7-38,88\%).

En el periodo analizado se publicaron artículos "strategy" y "strategic” casi todos los años, excepto en 2001, 2002 y 2007 para “strategy” y en 2001, 2002, 2003, 2010 y 2015 para “strategic”.

De los 28 artículos “strategy” los ejercicios en que se publicaron más artículos fueron 2006, 2008 y 2010 con 4 (14,28\%) artículos/año. De los 18 artículos “strategic” fueron 2009, 2013 y 2014 los años con mayor número de artículos publicados (3-16,66\%).

Ver los títulos de los artículos en Tabla 3 (“strategy”) y Tabla 4 (“strategic”).

\subsection{Resultados de los términos "public relations" y "PR" en las revistas de estrategia} 6.2.1. Revistas "strategy"

- Global Strategy Journal; Journal of Economics Management Strategy; y Journal of Family Business Strategy - No se identificó ningún artículo con los parámetros de búsqueda "public relations" o "PR" en el periodo analizado. 
- Business Strategy and the Environment - Se localizó un único artículo que cumplía los parámetros de búsqueda, publicado en 2009 y con autoría única, de género masculino. El artículo, titulado "Social and Environmental Reporting and the Corporate Ego", trata "on the results of a qualitative exploration into the target audiences for social and environmental reporting (SER)” y concluye que "broader stakeholders might be targeted with SER only insofar as doing so serves as a perceived endorsement of organizational communications, thereby embellishing the corporate ego’s fantasy of how it would like to be perceived” (Crawford, 2009: 254).

\subsubsection{Revistas "strategic"}

- Advances in Strategic Management-A Research Annual; Journal of Strategic Information Systems; International Journal of Strategic Property Management; Strategic Entrepreneurship Journal; y Strategic Organization - No se identificó ningún artículo con los parámetros de búsqueda "public relations" o "PR" en el periodo analizado.

- Strategic Management Journal - Se localizó un único artículo adaptado a los parámetros de búsqueda, publicado en 2003, con autoría doble y de género masculino. El artículo, titulado "Mapping moral philosophies: Strategic implications for multinational firms" relata, desde el punto de vista de los modelos éticos de toma de decisiones, como "Strategic managers appear increasingly under pressure from stakeholder concerns regarding social and ethical issues" (Robertson y Crittenden, 2003: 385). Ninguno de los autores es especialista en RP.

- Technology Analysis \& Strategic Management - Un único artículo, titulado "Meeting the challenge of aviation emissions: an aircraft industry perspective" cumplía los parámetros de búsqueda. Publicado en 2009, de autoría única y perteneciente al género masculino y, en el contexto del cambio climático, trata sobre el papel que juegan las emisiones de $\mathrm{CO}_{2}$ y $\mathrm{NO}_{\mathrm{x}}$ de los aviones y, tangencialmente, de la campaña de RP sobre la industria de la aviación impulsada por "green lobby groups, other NGOs and parts of the media” (Lawrence, 2009: 80). El autor no está especializado en RP.

\section{Discusión y conclusiones}

Los resultados de la investigación proporcionan respuesta a las preguntas de investigación: las revistas especializadas en RP son dos (2): Public Relations Review y Journal of Public Relations Research (PI1) y las especializadas en estrategia son once (11): Global Strategy Journal; Business Strategy and the Environment; Journal of Economics Management Strategy; Journal of Family Business Strategy; Advances in Strategic Management-A Research Annual; Journal of Strategic Information Systems; International Journal of Strategic Property Management; Strategic Entrepreneurship Journal; Strategic Organization; Strategic Management Journal; y Technology Analysis \& Strategic Management (PI2).

Se contempla abundantemente la perspectiva estratégica en los artículos de las revistas de RP (PI3), mientras que es prácticamente inexistente el tratamiento de las RP en las revistas de gestión especializadas en estrategia (PI4), ya que en estas últimas se han identificado únicamente 3 artículos, de los cuales 2 tratan de forma tangencial o anecdótica las RP. Se podría especular que, o bien la totalidad de los investigadores identificados prefieren publicar en las revistas de su campo, o bien que los editores de las revistas de gestión no se muestran proclives a aceptar a revisión artículos sobre temas principales ajenos a la gestión y/o la administración de empresas, según señalaba Tardy (2004: 250): "discourse patterns do not follow the expectations of the gatekeepers, they are more likely to be viewed as non-standard and to be excluded from publication (Bhatia, 1997 \& Kaplan, 2001)”. 
Los periodos temporales en que se han publicado un mayor número de artículos en las revistas especializadas en RP fueron los años 2012 (“strategy”, 16-15, 68\%) y 2014 (“strategic”, 18-17, 14\%) para Public Relations Review. Y los años 2006, 2008 y 2010 (“strategy” 4-14, 28\%) у 2009, 2013 у 2014 ("strategic” 3, 16,66\%) para Journal of Public Relations Research (PI5). En Public Relations Review no se publicó ningún artículo los años 2001 y 2006, y en Journal of Public Relations Research los años 2001, 2002, 2003, 2007, 2010 y 2015 (hasta el 15.07.15). La escasa presencia de artículos sobre RP en las once (11) revistas especializadas en estrategia no permiten extraer conclusiones significativas respecto a los periodos con mayor número de artículos publicados (PI6).

La distribución de género en Public Relations Review es de 92 (46,03\%) hombres y 109 (53,69\%) mujeres para "strategy"; y de 82 (39,23\%) hombres y 127 (60,76\%) mujeres para "strategic". En Journal of Public Relations Research es de 19 (38,00\%) hombres y 31 (62,00\%) mujeres para “strategy” y 24 (53,33\%) hombres y 21 (46,66\%) mujeres para "strategic”. Los autores de género femenino son prevalentes en el conjunto de autorías de estas dos revistas (excepto en el último caso), en contradicción con Castillo \& Carretón (2010: 310), quienes identificaron una supremacía de autorías masculinas en su estudio. Los datos tampoco son congruentes con la paridad de género de las revistas españolas estudiadas por Míguez, Baamonde \& Corbacho (2014), excepto en el último caso, donde sí se cumple (PI7). Tampoco en esta ocasión es posible extraer conclusiones significativas sobre el género de los autores de las once (11) revistas especializadas en estrategia debido a los escasísimos volúmenes publicados (PI8), aunque destacamos que la autoría masculina es total.

La prevalencia del número de autores por artículo en las revistas especializadas en RP es de 2 autores (48-47, 05\%) para "strategy” y de 1 autor (48-45, 71\%) para "strategic” en Public Relations Review y de 1 autor (13-46, 42\%) para "strategy” y 2 autores (7-38, 88\%) para “strategic” en Journal of Public Relations Research, en consistencia con Koehler (2001: 122) -1,8 autores en la década de 1990- y con Castillo \& Carretón (2010: 317) -2 autores en el 90,00\% de los casos- (PI9). Sobre el escaso número de autores (4) de los 3 escasos artículos de las 11 revistas especializadas en estrategia no es posible extraer conclusiones significativas (PI10).

Los títulos de los artículos de las revistas especializadas en RP aparecen consignados en la quinta columna de las Tablas 1, 2, 3 y 4 (PI11) y los de los artículos de las revistas especializadas en estrategia se describen en la quinta columna de las Tablas 5 y 6 (PI12).

El análisis bibliométrico muestra que el tratamiento de la estrategia aplicada a las RP es muy abundante en las dos revistas especializadas en RP, lo que confirma la literatura de referencia con respecto al interés mostrado por los autores de la muestra por la función directiva de los profesionales de las RP al frente de un departamento independiente (Prat Gaballí, 1958; Matrat, 1970: 27, 37-49; Arnaldi, 1971: 217; Ugeux, 1973: 32-33; Ledigham \& Bruning, 2000; Van Ruler, 2000; Van Ruler y otros 2000, 2004; Van Ruler \& Verčič, 2002, 2004) y, por consiguiente, sobre sus límites funcionales y su nivel de responsabilidad en el diseño y en la ejecución del plan estratégico de comunicación y su contribución al plan estratégico corporativo; sobre el proceso de rendición de cuentas y a quien rendirlas jerárquicamente; así como su posible integración en el comité de dirección, en consistencia con los resultados de los estudios ECM-European Communication Monitor (2011) y State of the Profession Survey (2014).

Sin embargo, el interés mostrado por los autores de las revistas de gestión especializadas en estrategia apunta en la dirección contraria, ya que no se observa que el debate en torno al departamento de RP y a las funciones y responsabilidades de sus directivos, desde cualquiera de sus posibles perspectivas por lo que respecta a la administración de empresas en su sentido más amplio, tenga una correspondencia similar en los escasísimos, por no decir casi inexistentes artículos identificados. 
Asimismo, el interés por otras posibles orientaciones temáticas de las RP de ámbito más general y no centrado exclusivamente en la gestión de la comunicación se constata, también, casi nulo (a excepción, quizás, de Crawford, 2009) y aunque la escasez de resultados no permite extraer conclusiones significativas desde un punto de vista estadístico, señalamos que, en base a las evidencias empíricas, las RP no se constituyen en ningún caso en el tema principal tratado, lo cual puede tener su lógica en que ninguno de los autores de los artículos es especialista en ese campo.

En futuras investigaciones se pretenden realizar estudios metodológicamente idénticos, con muestras de estudio más amplias, como Scopus o Latindex, RESH e IN-RECS (*) -en lengua no-inglesa (Van Leeuwen, 2001; Yukio, 1992)-, para obtener evidencias y desarrollar, posteriormente, sucesivos estudios comparativos e identificar, si las hubiere, posibles desviaciones producto de la diversidad lingüística (Tardy, 2004).

(*) N. del E.-- IN-RECS, realizado por el grupo EC ${ }^{3}$, de la Universidad de Granada, ha dejado de funcionar hace pocos años.

\section{Referencias bibliográficas}

Arnaldi, P. (1971). Manual de Relaciones Públicas. Madrid: Hispano Europea (2ª ed.).

Beniger, J.R. (1990). Who are the most important theorists of communication? Communication Research, 17, p. 698-715.

Bornmann, L., Mutz, R., Neuhaus, C., \& Daniel, H.-D. (2008). Citation counts for research evaluation: Standards of good practice for analyzing bibliometric data and presenting and interpreting results. Ethics in Science and Environmental Politics, 8, p. 93-102.

Brody, T., Carr, L., Gingras, Y., Hajjem, Ch., Harnad, S., \& Swan, A. (2007). Incentivizing the open access research Web: Publication-archiving, data-archiving and scientometrics. CT Watch Quarterly, 3.

Bunz, U. (2005). Publish or perish: A limited author analysis of ICA and NCA journals. Journal of Communication, 55, p. 703-720.

Burnham, J.C. (1990). The evolution of editorial peer review, Journal of the American Medical Association, 263 (9 March).

Butler, L. (2008). Using a balanced approach to bibliometrics: Quantitative performance measures in the Australian research quality framework. Ethics in Science and Environmental Politics, 8, p. 8392.

Cáceres, M.D., \& Caffarel, C. (1993). “La comunicación en España: planteamientos temáticos y metodológicos entre 1987 y 1990”. En AICE, La investigación en la comunicación (pp. 23-30). III Simposio de la Asociación de Investigadores en Comunicación del Estado Español (AICE), Madrid.

Campell, PH. (2008). Escape from the impact factor. Ethics in Science and Environmental Politics, 8, p. 5-7.

Castillo, A., \& Carretón, $M^{a}$ C. (2010). Investigación en Comunicación. Estudio bibliométrico de las revistas de Comunicación en España. Comunicación y Sociedad, 13(2), p. 289-327. 
Castillo, A., \& Rubio, A. (2010). "La calidad de indización en las bases de datos científicas sobre Comunicación. Estudio comparativo de las revistas con mayor índice de impacto según el ISI del Journal Citations Reports”, Congreso Comunicación y Desarrollo en la Era Digital, 3- 5 febrero 2010, Málaga.

Castillo, A., \& Xifra, J. (2006). Investigación bibliométrica de las tesis doctorales españolas sobre Relaciones Públicas, Anàlisi, 34, p. 141-161.

Castillo-Esparcia, A., Rubio-Moraga, A., \& Almansa-Martínez, A. (2012). La investigación en Comunicación. Análisis bibliométrico de las revistas de mayor impacto del ISI. Revista Latina de Comunicación Social, 67, p. 248-270.

Chaumely, J., \& Huisman, D. (1963). Les Relations Publiques. París: P.U.F.

Clarke, R. (2007). The cost profiles of alternative approaches to journal publishing. First Monday, 12(12), (December).

Compte-Pujol, M., De Urquijo, B., \& Matilla, K. (2016). La investigación en marcas de territorio y diplomacia pública en España. Un estudio bibliométrico de las revistas científicas españolas especializadas en Comunicación indexadas en Latindex (1980-2016). Anales de Documentación, 19(2), p. 1-53.

Cope, B., \& Kalantzis, M. (1993). "Introduction: How a Genre Approach to Literacy can Transform the Way Writing is Taught”. En B. Cope \& M. Kalantzis (eds.), The Powers of Literacy: A genre approach to teaching writing (pp. 1-21). London: Falmer Press.

Cope, B., \& Kalantzis, M. (2009). Signs of Epistemic Disruption: Transformations in the Knowledge System of the Academic Journal. First Monday, 14(4, 06.04.09, [s.n.], 2009.

Cope, B., \& Kalantzis, M. (2010). Evaluating Webs of Knowledge: A Critical Examination of the 'Impact Factor'. Logos: The Journal of the World Book Community, 21, p. 117-132.

Cope, B., Kalantzis, M. \& Magee, L. (2011). Towards a Semantic Web: Connecting Knowledge in Academic Research. Cambridge, UK: Woodhead Publishing.

Cope, B., \& Phillips, A. (eds.) (2013). The Future of the Academic Journal. Oxford, UK: Chandos, $2^{\mathrm{a}}$ ed.

Craig, I.D., \& Ferguson, L. (2009). "Journals ranking and impact factors: How the performance of journals is measured”. En: B. Cope \& M. Kalantzis (eds). The future of the academic journal. Oxford, UK: Chandos, $2^{\text {a }}$ ed.

Cutlip, S. (1995). Public Relations History: From the $17^{\text {th }}$ to the $20^{\text {th }}$ Century. The Antecedents. New Jersey, NJ: Lawrence Erlbaum.

Davies, J.E. (2009). "Libraries and the future of the journal: Dodging the crossfire in the erevolution; or leading the charge?”. En: B. Cope \& A. Phillips (eds). The future of the academic journal. Oxford, UK: Chandos, $2^{\mathrm{a}}$ ed.

De Solla Price, D.J. (1965). Networks of Scientific Papers. The pattern of bibliographic references indicates the nature of the scientific research front. Science, 149, 30 july, p. 510-515.

Dewatripont, M., Ginsburgh, V., Legros, P., \& Walckiers, A. (2006). Study on the economic and technical evolution of the scientific publication markets in Europe. Brussels: European Commission. 
Edlin, A.S., \& Rubinfeld, D.L. (2004). Exclusion or efficient pricing? The 'big deal' bundling of academic journals, Berkeley, CA: University of California.

Evans, J.A. (2008). Electronic publication and the narrowing of science and scholarship, Science, 321(5887), 18 Julio), p. 395-399.

Ferreira, J., Fernandes, C., \& Ratten, V. (2016). A co-citation bibliometric analysis of strategic management research. Scientometrics, 109(1), p. 1-32.

Fink, J.L., \& Bourne, (2007). Ph.E. Reinventing scholarly communication for the electronic age, CTWatch Quarterly, 3.

Fusell Sisco, H., Pressgrove, G., \& Collins, E.L. (2013). Paralleling the Practice: An Analysis of the Scholarly Literature in Nonprofit Public Relations, Journal of Public Relations Research, 25(4), p. 282-306.

García-Guinea, J., \& Sota Rius, J., de la. (1998). Las consecuencias de publicar en revistas científicas escritas en español en España, Interciencia, 23(3), p. 185-187.

Ginsparg, P. (2007). Next-generation implications of open access. CT Watch Quarterly, 3.

Goldman, E.F. (1948). Two-Way Street: The Emergence of the Public Relations Counsel. Boston, MA: Bellman.

González Quirós, J.L., \& Martín, K.G. (2009). “Arguments for an open model of science”. En B. Cope \& A. Phillips (eds). The future of the academic journal. Oxford, UK: Chandos, $2^{\mathrm{a}}$ ed.

Guedón, J.-C. (2001). In Oldenburg's long shadow: Librarians, research scientists, publishers, and the control of scientific publishing. Association of Research Libraries.

Hannay, T. (2007). Web 2.0 in science. CT Watch Quarterly, 3.

Harnad, S. (2008). Validating research performance metrics against peer rankings. Ethics in Science and Environmental Politics, 8, p. 103-107.

Harnad, S. (2009). “The Post-Gutenberg open access journal”. En B. Cope \& A. Phillips (eds.), The future of the academic journal. Oxford, UK: Chandos, $2^{\mathrm{a}}$ ed.

Harzing, A.-W. K., \& Van der Wal, R. (2008). Google Scholar as a new source for citation analysis. Ethics in Science and Environmental Politics, 8, p. 61-73.

Jakubowicz, A. (2009). Beyond the static text: Multimedia interactivity in academic publishing. En: B. Cope \& A. Phillips (eds.), The future of the academic journal. Oxford, UK: Chandos, $2^{\mathrm{a}}$ ed.

Jefferson, T., Wager, E., \& Davidoff, F. (2002). Measuring the quality of editorial peer review. Journal of the American Medical Association, 287, p. 2786-2790.

Jones, D.E. (1997). Investigació sobre comunicació social a l'Espanya de les autonomies. Anàlisi, 21, p. 101-120.

Judson, H.F. (1994). Structural transformations of the sciences and the end of peer review. Journal of the American Medical Association, 272, p. 92-94.

Ki, E.-J., \& Shin; J.-H. (2006). Status of organization-public relationship research from an analysis of published articles, 1985-2004. Public Relations Review, 32(2), p. 194-195. 
Kim S.-Y., Choi, M.-I., Reber, B.H., \& Kim, D. (2014). Tracking public relations scholarship trends: Using semantic network analysis on PR Journals from 1975 to 2011. Public Relations Review, 40(1), p. 116-118.

Koehler, W. (2001). Information science as 'Little Science': The implications of a bibliometric analysis of the Journal of the American Society for Information Science. Scientometrics, 51(1), p. 117-132.

Kousha, K., \& Thelwall, M. (2007). Google Scholar citations and Google Web/URL citations: A multi-discipline exploratory analysis. Journal of the American Society for Information Science and Technology, 5(7), p. 1055-1065.

Krippendorff, K. (2004). Content Analysis: An Introduction to Its Methodology. Thousand Oaks, CA: Sage, $2^{\text {a }}$ ed.

Lauf, E. (2005). National diversity of major international journals in the field of communication. Journal of Communication, 55(1), p. 139-151.

Ledingham, J.A., \& BruningR, S.D. (2000). Public Relations as Relationship Management. A Relational Approach to the Study and Practice of Public Relations, Mahwah, NJ: Lawrence Erlbaum.

Lee, K., \& Bero, L. (2006). What authors, editors and reviewers should do to improve peer review. Nature.

Lievrow, L.A. (1990). "Reconciling structure and process in the study of scholarly communication”. En C. L. Borgman (Ed.), Scholarly Communication and Bibliometrics (pp. 59-72). Newbury Park, CA: Sage.

López Baena, A.J., Valcárcel Cases, M., \& Barbancho Medina, M. (2005). Propuesta de un sistema de evaluación de revistas científicas en las áreas de ciencias humanas y sociales. Revista Española de Documentación Científica, 28(1), p. 222-248.

López Piñero, J.M. (1972). El análisis estadístico y sociométrico de la literatura científica. Valencia: Centro de Documentación e Informática Médica de la Facultad de Medicina.

Mabe, M.A., \& Amin, M. (2002). Dr. Jekyll and Dr. Hyde: Author-reader asymmetries in scholarly publishing. Aslib Proceedings, 54, p. 149-157.

Marca-Francés, G., Matilla, K., \& Ortiga-Fontgivell, B. (2014). Las revistas académicas de comunicación en salud y de relaciones públicas: un análisis bibliométrico comparativo. En K. Matilla (Coord.), Cambio social y Relaciones Públicas (pp. 87-100). Barcelona: UOC.

Martínez Nicolás, M. (2009). La investigación sobre comunicación en España. Evolución histórica y retos actuales. Revista Latina de Comunicación Social, 64.

Martínez Nicolás, M. \& Saperas-Lapiedra, E. (2016). Objetos de estudio y orientación metodológica de la reciente investigación sobre comunicación en España (2008-2014): Análisis de los trabajos publicados en revistas científicas españolas. Revista Latina de Comunicación Social, 71, p. 1.3651.384 .

Matilla, K., Oliveira, A., \& Compte-Pujol, M. (2015). Strategy and Public Relations in Europe during the $21^{\text {st }}$ Century: Is there a gap between praxis and academia? Wulfenia Journal, 2(22), feb.

Matrat, L. (1970). Relations Publiques et Management. Bruxelles: CERP. 
McCabe, M.J., Nevo, A., \& Rubinfeld, D.L. (2006). The pricing of academic journals. Berkeley, CA: University of California.

McNutt, M. (2014). Be one of the first. Science, 19 September, 345(6203).

Meho, L.I. (2007). The rise and rise of citation analysis. Physics World, 20, p. 32-36.

Míguez-González, M., Baamonde-Silva, X.M., \& Corbacho-Valencia, J.M. (2014). A bibliographic study of public relations in Spanish media and communication journals, 2000-2012, Public Relations Review, 40(5), p. 818-828.

Míguez-González, M., Corbacho-Valencia, \& J., Baamonde-Silva, X. (2016). Tendencias de investigación sobre relaciones públicas en revistas internacionales: el caso del 'Journal of Public Relations Research’ 2012-2014. Revista Internacional de Relaciones Públicas, 12(6), p. 5-24.

Moloney, K. (2000). Rethinking Public Relations. The Spin and the Substance. London, UK: Routledge.

Morris, S. (2009). "The tiger in the corner: Perhaps journals will not be central to the lives of tomorrow's scholars?”. En B. Cope \& A. Phillips (eds). The future of the academic journal. Oxford, UK: Chandos, $2^{\mathrm{a}}$ ed.

Nessmann, K. (1995). Public Relations in Europe: a comparison with the United States. Public Relations Review, 21(2), p. 151-160.

Norris, M., \& Oppenheim, Ch. (2007). Comparing alternatives to the Web of Science for coverage of the social sciences' literature. Journal of Informetrics, 1(2), p. 161-169.

Noyons, E.C.M., Moed, H.F., \& Luwel, M. (1999). Combining mapping and citation analysis for evaluative bibliometric purposes: A bibliometric study. Journal of the American Society for Information Science, 50(2), p. 115-131.

Osca-Lluch, J., Velasco, E., López, M., \& Haba, J. (2009). Co-authorship and citation networks in Spanish history of science research, Scientometrics, 80(2), p. 373-385.

Paisley, W. (1989). Bibliometrics, scholarly, communication and communication research. Communication Research, 16, p. 701-717.

Pasadeos, Y., \& Renfro, B. (1989). A citation study of public relations research 1975-1986. Public Relations Review, 15(3), p. 48-50.

Pasadeos, Y., \& Renfro, B. (1992). A bibliometric analysis of public relations research. Journal of Public Relations Research, 4(3), p. 167-187.

Pasadeos, Y., Renfro, B. \& Hanily, M. (1999). Influential authors and works of public relations scholarly literature: A network of recent research. Journal of Public Relations Research, 11(1), p. 29-52.

Pasadeos, Y., Berger, B., \& Renfro, B. (2010). Public relations as a maturing discipline: An update on research networks. Journal of Public Relations Research, 22(2), p. 136-158.

Pauly, D., \& Stergiou, K.I. (2008). Re-interpretation of 'influence weight' as a citation-based Index of New Knowledge (INK). Ethics in Science and Environmental Politics, 8, p. 75-78.

Prat Gaballí, P. (1958). "Prefacio”. En W.A. Nielander y R.W. Miller, Relaciones Públicas. Cómo Método para crear prestigio y promover ventas. Barcelona: Hispano Europea. 
Pritchard, A. (1969). Statistical Bibliography or Bibliometrics? Journal of Documentation, 25(4), p. 348-349.

Roca-Correa, D., \& Pueyo-Ayhan, N. (2012). La productividad científica en Comunicación a través de la revista 'Zer'. Revista Latina de Comunicación Social, 67, p. 292-321.

Sallot, L.M., Lyon, L.J., Acosta-Alzuru, C., \& Jones, K.O. (2003). From aardvark to zebra: A new millennium analysis of theory development in public relations academic journals. Journal of Public Relations Research, 15(1), p. 27-90.

Schroeder, R. (2007). Pointing users toward citation searching: Using Google Scholar and Web of Science. Libraries and the Academy, 7, p. 243-248.

Seon-Kyoung, A., \& I-Huei, CH. (2012). "Crisis Communication Research in Public Relations Journals. Tracking Research Trends Over Thirty Years”. En T. Coombs \& S. J. Holladay (Eds.), The Handbook of Crisis Communication (65-90). Chichester, UK: Wiley-Blackwell.

Simons, K. (2008). The misused impact factor. Science, 322(5899) (10 October), 165.

So, C. (1988). Citation patterns of core communication journal: An assessment of the development status of communication. Human Communication Research, 15(2), p. 236-255.

Stanley, CH.A. (2007). When counter narratives meet master narratives in the journal editorialreview process. Educational Researcher, 36, p. 14-24.

Suber, P. (2007). Trends favoring open acces. CT Watch Quarterly, 3.

Tardy, C. (2004). The role of English in scientific communication: lingua franca or Tyrannosaurus rex? Journal of English for Academic Purposes, 3(3), p. 247-269.

Tenopir, C., \& King, D.W. (2009). “The growth of journals publishing”. En B. Cope \& A. Phillips (eds). The future of the academic journal. Oxford, UK: Chandos, $2^{\mathrm{a}}$ ed.

Todd, P.A., \& LADLE, R.J. (2008). Hidden dangers of a 'citation culture'. Ethics in Science and Environmental Politics, 8, p. 13-16.

Ugeux, W. (1973). Les relations publiques. Verviers, France: Gérard Marabout.

Van de Sompel, H., \& Lagoze, C. (2007). Interoperability for the discovery, use, and re-use of units of scholarly communication. CT Watch Quarterly, 3.

Van Leeuwen, T.N., Moed, H.F., Tijssen, R.J.W., Visser, M.S., \& Van Raan, A.F.J. (2001).

Language biases in the coverage of the Science Citation Index and its consequences for international comparisons of national research performance, Scientometrics, 51(1), p. 335-346.

Van Ruler, B. (2000). “Dimensions of European Public Relations”, Public Relations World Congress 2000, October 22-24, Chicago, ILL.

Van Ruler, B., \& Vercic, D. (2004). Public relations and communication management in Europe. Berlin: Mouton de Gruyter.

Van Ruler, B., \& Vercic, D. (2002). “The Bled Manifesto on Public Relations”. $9^{\text {th }}$ International Public Relations Research Symposium, Bled. Lubljana (Eslovenia), 4-5 July.

Van Ruler, B., Vercic, D., Bütschi, G., \& Flodin, B. (2000). The European Body of Knowledge on 
Public Relations. Communication Management'; 'The Report of the Delphi Research Project 2000. European Association for Public Relations Education and Research, June.

Wilbanks, J. (2007). Cyberinfrastructure for knowledge sharing. CT Watch Quarterly, 3.

Willinsky, J. (2005). The access principle: The case for open research and scholarship. Cambridge, MASS: MIT Press.

Wimmer, R.D., \& Dominick, J.R. (1996). La investigación científica de los medios de comunicación: una introducción a sus métodos. Barcelona: Bosch Comunicación.

Xifra, J., \& Castillo, A. (2006). Forty Years of Doctoral Public Relations Research in Spain: A Quantitative Study of Dissertation Contribution to Theory Development, Public Relations Review, 32, p. 302-308.

Yukio, T. (1992). The Dominance of English and Linguistic Discrimination. Media Development, 15(1), p. 32-34.

\section{Cómo citar este artículo / Referencia normalizada}

M Compte-Pujol, K Matilla, S Hernández (2018): "Estrategia y Relaciones Públicas: un estudio bibliométrico comparativo". Revista Latina de Comunicación Social, 73, pp. 748 a 764.

http://www.revistalatinacs.org/073paper/1280/39es.html

DOI: $10.4185 /$ RLCS-2018-1280

- En el interior de un texto:

...M Compte-Pujol, K Matilla, S Hernández (2018: 748 a 764) ...

o

...M Compte-Pujol et al, 2018 (748 a 764) ...

Artículo recibido el 18 de enero de 2018. Aceptado el 15 de abril. Publicado el 19 de abril de 2018 